\title{
Diagnostic Yield of Fluoroscopy-Guided Biopsy for Infectious Spondylitis
}

\author{
B.J. Kim, J.W. Lee, S.J. Kim, G.Y. Lee, and H.S. Kang
}

\begin{abstract}
BACKGROUND AND PURPOSE: CT is currently the method of choice for guiding biopsy of lesions of the spine. However, in our hospital, fluoroscopy-guided percutaneous biopsy has been preferred for several years because of equipment availability and easy craniocaudal angulation. The aim of this study was to evaluate the efficacy of fluoroscopy-guided percutaneous biopsy in a clinical setting for diagnosing infectious spondylitis.
\end{abstract}

MATERIALS AND METHODS: A retrospective study was performed to evaluate 170 fluoroscopy-guided percutaneous biopsies in 140 patients (male/female = 70:70; mean age, 65.1 years; range, 16-89 years) in a clinical setting who were suspected of having infectious spondylitis between July 2003 and March 2010. Diagnosis was based on pathologic confirmation by tissue or culture from biopsy. The percentage of adequate specimens for diagnosis, histopathologic diagnosis for infectious spondylitis, and positive cultures for causative organisms were evaluated by retrospective review of medical records.

RESULTS: Adequate specimens for diagnosis were obtained in 165 of 170 cases (97.1\%). The diagnosis of infectious spondylitis resulted in 134 of 170 cases confirmed through histopathology or clinical outcome (78.8\%). In 51 of 134 cases (38.1\%), the causative organism was confirmed by specimens from percutaneous bone biopsy. There were no biopsy-related major complications. The most common organism isolated was Mycobacterium tuberculosis (24 cases), followed by Staphylococcus aureus, Streptococcus agalactiae, and Streptococcus viridans.

CONCLUSIONS: Fluoroscopy-guided percutaneous biopsy is as accurate and effective as CT-guided biopsy for diagnosing infectious spondylitis.

ABBREVIATION: TB PCR $=$ Tuberculosis polymerase chain reaction

I

nfectious spondylitis is a rare disease that may cause severe complications such as irreversible neurologic defects and vertebral deformity. ${ }^{1}$ The diagnosis of infectious spondylitis is supported by laboratory data and appropriate imaging changes in investigations such as plain radiography, CT, and MR imaging. For correct diagnosis and optimal antimicrobial treatment, isolation of the causative organism and histologic diagnosis are necessary. ${ }^{2}$ To achieve these, a spinal biopsy to get sufficient tissue for identification of the causative organism is essential.

Gradually through the years, image-guided percutaneous biopsy has replaced open biopsy because it has many advantages, including cost and time, lower morbidity, avoidance of general

Received December 16, 2011; accepted after revision February 27, 2012.

From the Department of Radiology (B.J.K., J.W.L., G.Y.L., H.S.K.), Seoul National University Bundang Hospital, Gyeonggi-do, Korea; and Department of Radiology (S.J.K.), Boramae Medical Center, Seoul, Korea.

Please address correspondence to Joon Woo Lee, MD, Department of Radiology, Seoul National University Bundang Hospital, 300 Gumidong, Bundang-Gu, Seong Nam, Gyeongi-do, 463-707 Korea; e-mail: joonwoo2@gmail.com

http://dx.doi.org/10.3174/ajnr.A3120 anesthesia, and outpatient procedure availability. ${ }^{3}$ Of all the imaging methods, CT is currently the method of choice for guiding biopsy of lesions of the spine for many authors. ${ }^{4-7}$ However, in our hospital, fluoroscopy-guided percutaneous biopsy has been preferred for several years because of equipment availability and ease of craniocaudal angulation. In our experience, fluoroscopic guidance is as appropriate as CT guidance for vertebral body biopsy to diagnose infectious spondylitis.

There have been few large studies of fluoroscopy-guided percutaneous biopsy for infectious spondylitis, and compared with a high accuracy rate for confirmation of neoplastic spinal lesions, ${ }^{3,7-9}$ percutaneous spinal biopsy for bacteriologic diagnosis has a variable rate of reported success in patients with spondylitis. ${ }^{10,11}$ Even in more recent studies, ${ }^{12,13}$ the identification of a causative organism proved impossible in a number of cases, and empiric antibiotic therapy had to be used.

The aim of this study was to evaluate the efficacy of fluoroscopy-guided percutaneous biopsy in a clinical setting for diagnosing infectious spondylitis. 


\section{MATERIALS AND METHODS}

\section{Study Population}

The retrospective study was approved by our institutional review board. Informed consent was waived. The materials reviewed by the authors for this study were medical records, including outpatient and emergency department notes, admission notes, surgical reports, radiology reports, pathology reports, and microbiology laboratory results. Between July 2003 and March 2010, fluoroscopy-guided percutaneous spine biopsies were performed for 309 patients at our institution. Of these, we selected patients in whom the fluoroscopy-guided biopsy was performed to confirm or rule out the clinical or radiologic possibility of infectious spondylitis. Patients who underwent biopsy for suspected primary bone tumors or metastases were excluded.

In our institution, in principle, empiric antibiotic treatment was not started until the culture result was reported. Re-biopsy was indicated if results were negative at 1-week follow-up. After re-biopsy, empiric antimicrobial treatment was started. A total of 170 biopsy procedures were performed in 140 patients with suspected infectious spondylitis.

The study group consisted of 70 males and 70 females, with ages ranging from 16 to 89 years.

One hundred fourteen patients underwent only 1 procedure. In 26 of 140 patients, re-biopsies were performed to identify the causative organism for treatment of infectious spondylitis: Twenty-six patients underwent 2 biopsies, and 2 patients underwent 3 biopsies. However, because the time interval between the first biopsy procedure and successive biopsies in these 2 patients was $>6$ months, we regarded these cases as 2 separate events and these 28 patients were considered as 56 re-biopsy cases. In 22 of the 26 patients in whom multiple procedures were performed, the procedures were performed at the same levels; the rest were performed at the different levels. Lesions were located in the following regions (Table 1): 1 cervical, 22 thoracic, and 147 lumbar.

Diagnosis of infectious spondylitis was defined when the causative organism was identified from spinal biopsy, or the clinical course satisfied 1 of the follow conditions: confirmation by operation, improvement after empiric antibiotic treatment, identification of the causative organism by other procedures such as blood culture, or aggravation on follow-up MR imaging.

\section{Technique}

In all cases, percutaneous biopsy was performed to obtain a specimen for culture and histologic examination under fluoroscopic guidance as described in a previous study. ${ }^{14}$ In all cases, bacterial culture, TB PCR, staining for acid-fast bacilli, fungal culture, and histologic examination were performed. The method of approach depended on the lesion location. The anterior approach was used for cervical spine lesions; an intercostovertebral or transpedicular approach, for the thoracic spine; and a transpedicular approach, for lumbar lesions. Immediately before the biopsy, complete blood count, activated partial thromboplastin time, and prothrombin time were measured. All patients were monitored during the procedure with a pulse oximeter, an electrocardiographic monitor, and an automated blood pressure cuff.

Percutaneous spinal biopsy was performed under the guidance of a biplane (Integris Allura 12/12; Philips Healthcare, Best,
Table 1: Level in bone biopsy

\begin{tabular}{lr}
\hline \multicolumn{1}{c}{ Level } & No. \\
\hline Cervical & 1 \\
spine & \\
Thoracic & 22 \\
spine & \\
Lumbar spine & 147 \\
Total & 170 \\
\hline
\end{tabular}

the Netherlands) or uniplane (Integris Allura Xper FD 20; Philips Healthcare) DSA unit. All biopsies were performed with a 15-ga Bonopty biopsy needle system (RADI Medical Systems, Uppsala, Sweden). The Bonopty needle has the advantage of obtaining an adequate amount of specimen for diagnosis as well as facilitating needle positioning compared with other needles, so it was used in the study despite the high cost. Each percutaneous biopsy was performed more than twice to obtain sufficient tissue and aspiration material to identify the causative organism. Also, in 22 cases, disk aspiration was performed with spinal biopsy, and epidural or paravertebral abscess aspiration was performed in 7 cases.

\section{Clinical Outcome}

One radiologist reviewed the microbiologic and histologic reports to investigate the adequacy or inadequacy of the specimen for analysis, the rate at which infectious spondylitis was confirmed through the microbiologic and histologic reports or clinical information, the rate at which causative organisms were isolated in infectious spondylitis, and the kind of commonly isolated organisms. The radiologist also investigated the rebiopsy results separately to ascertain the rate and the cause of the positive re-biopsy after negative results on the first biopsy.

\section{RESULTS}

In all cases, there were no technical failures such as inaccessibility to the lesion or biopsy at the wrong location while obtaining the tissue. There were no biopsy-related major complications such as hemorrhage, infection, sinus tract formation, fracture, or pneumothorax. An adequate amount of specimen for diagnosis was obtained in 165 cases $(97.1 \%)$, with only 5 cases yielding inadequate specimens. Of these 5 cases, re-biopsy was performed in 1 case. In the other 4 cases, re-biopsy was not performed due to death by aspiration pneumonia (1 case), surgery for symptom aggravation (1 case), and spontaneous improvement ( 2 cases). The diagnosis of infectious spondylitis resulted in 134 of 170 cases confirmed through histopathology or clinical outcome (78.8\%) (Tables 2 and 3). In 51 of 134 cases (38.1\%), the causative organism was isolated by specimens from percutaneous bone biopsy. The isolated causative organisms were as follows: Mycobacterium tuberculosum (26 cases) was the most frequent organism, followed by Staphylococcus aureus (13 cases), Streptococcus agalactiae (4 cases), Streptococcus viridans (2 cases), coagulase-negative Staphylococcus organisms, Escherichia coli, Enterococcus faecalis, Enterobacter cloacae, Staphylococcus epididymis, and Klebsiella organisms (1 case each).

Re-biopsy was performed in 26 patients comprising 52 cases. Of these, the causative organism was identified in re-biopsy after a negative first biopsy result in only 2 patients. These were $M$ tuberculosum and $S$ aureus. In 6 patients, the causative organism was 
Table 2: The classification of the case by pathologic report ${ }^{\mathrm{a}}$

\begin{tabular}{cr} 
& No. \\
\hline Histology(+) & 115 \\
Culture(+) & 51 \\
Culture(-) & 64 \\
Histology(-) & 55 \\
Infection & 32 \\
Noninfection & 13 \\
Follow-up loss & 1 \\
Inadequate specimen & 5 \\
Etc & 4 \\
Total & 170 \\
\hline
\end{tabular}

Note:-Etc. indicates the case that infectious spondylitis was suspected on MRI image, but clinical symptoms were improved without empirical antibiotic treatment. a For Histology (+), the pathology report was recorded as follows: "consistent with infectious spondylitis," "active or acute inflammation," "suggestive of osteomyelitis," "chronic granulomatous inflammation." Histology $(-)$ is the rest except the histology $(+)$. Culture $(+)$ indicates cases of identification of the causative organism. Culture $(-)$ is cases of no identification of the causative organism.

\begin{tabular}{|c|c|c|}
\hline & $\begin{array}{l}\text { Histology(+), } \\
\text { Culture(-) }\end{array}$ & Culture(- \\
\hline Infection & 53 & 30 \\
\hline Confirmed by operation & 13 & 4 \\
\hline $\begin{array}{l}\text { Isolation of the causative organism } \\
\text { by blood culture }\end{array}$ & 12 & 5 \\
\hline $\begin{array}{l}\text { Improvement after empiric antibiotic } \\
\text { treatment }\end{array}$ & 25 & 19 \\
\hline Aggravation on follow-up MRI & 3 & 1 \\
\hline Noninfection & 11 & 20 \\
\hline Compression fracture & 6 & 7 \\
\hline Degenerative change (Modic type I) & 2 & 3 \\
\hline Hemorrhage by previous trauma & 0 & 2 \\
\hline $\mathrm{CML}$ & 0 & 1 \\
\hline Follow-up loss & 1 & 1 \\
\hline \multirow[t]{2}{*}{ Etc } & 2 & 6 \\
\hline & 64 & 50 \\
\hline
\end{tabular}

Note:-Etc. indicates the case that infectious spondylitis was suspected on MRI image, but clinical symptoms were improved without empirical antibiotic treatment; CML, chronic myelomonocytic leukemia.

a For Histology (+), the pathology report was recorded as "consistent with infectious spondylitis, "active or acute inflammation," "suggestive of osteomyelitis," and "chronic granulomatous inflammation." Histology $(-)$ indicates the rest except the histology $(+)$. Culture $(+)$ is identification of the causative organism. Culture $(-)$ is no identification of the causative organism.

identified in both the first and second biopsy, and these cases were all due to $M$ tuberculosum spondylitis. This result was due to a delay in the report for the TB PCR after the first biopsy, when only the TB PCR was positive. In the remaining 18 patients, the causative organism could not be identified in both the first and second biopsies. Of these, 12 patients were diagnosed as having infectious spondylitis, and 4 patients were diagnosed with conditions due to noninfectious causes such as compression fracture or degenerative change.

\section{DISCUSSION}

Infectious spondylitis accounts for $2 \% \sim 4 \%$ of cases of skeletal infection. ${ }^{15}$ However, there is evidence that the incidence is rising due to longer life expectancy for patients with chronic debilitating disease, immunosuppressive therapy, increasing use of indwelling devices, and spinal surgery. ${ }^{2,16,17}$ Because delays in diagnosis can result in more severe outcomes, ${ }^{18}$ early diagnosis and prompt application of appropriate antibiotic therapy for cultured organ- isms are crucial for successful nonsurgical treatment of infectious spondylitis and prevention of further morbidity. ${ }^{19,20}$

Percutaneous needle biopsy of the spine was introduced by Robertson and Ball in $1935 .^{21}$ It is a less invasive procedure than open biopsy. However, because of the relatively small amount of tissue obtained and the amount of normal tissue damaged by a blind approach, the clinical validity of this procedure was limited. To improve both clinical validity and safety, better access routes and guidance methods have been developed for more accurate access to involved vertebrae, as well as improvements in needle design. $^{22,23}$

Since the introduction of fluoroscopic guidance by Siffert and Arkin, ${ }^{24}$ radiographic guidance of the needle has become routine for vertebral bone biopsy, especially after the advent and popularization of the multidirectional image intensifier. ${ }^{25,26}$ In 1981, Adapson et $\mathrm{al}^{27}$ described the use of a CT scan for percutaneous biopsy.

Although currently many radiologists use CT to guide percutaneous biopsy, we prefer to use fluoroscopic guidance when we suspect infectious spondylitis in our institution. We think that the advantages of fluoroscopic guidance are the following: 1) a wide range of craniocaudal angulation, 2) easy perception of the needle position in the vertebral body in the lateral view, 3) short procedure time, and 4) real-time visualization and control of the needle position.

The most common area for infectious spondylitis is in a vertebral body near the disk space. In cases of L5-S1 involvement, a biopsy will target the L5 lower vertebral body rather than the L5/S1 disk or S1 upper vertebral body because bone marrow yields a more adequate specimen than disk and targeting the S1 upper body is difficult due to the overlying shadow of the iliac bones. However, targeting the L5 lower vertebral body requires a large cranial angulation, and for this reason, fluoroscopy is more convenient than $\mathrm{CT}$ in these cases. In early infectious spondylitis, the involved area cannot be detected by CT until the osteolytic area is evident, so biopsy targeting is achieved by MR imaging. A lateral view is needed on MR imaging to introduce a needle into the correct area of the abnormal vertebral body. In contrast, CTguided biopsy is done on the axial plane, so the needle position is corrected by comparison with a scout image. With fluoroscopy, real-time correction and confirmation of the needle position can be attained with a lateral view by matching the targeted area on the MR sagittal image. In our experience, fluoroscopic guidance can be easily performed with a short procedure time.

In our study, adequate specimens were obtained by percutaneous biopsy in 165 of 170 cases $(97.1 \%)$. Our results are comparable with those in other large studies of CT guidance. ${ }^{3,7,9}$ Our results are also consistent with those of Nourbakhsh et al, ${ }^{28}$ who reported that studies involving the use of CT demonstrated slightly higher rates for adequacy $(92.6 \%$ compared with $90.1 \%$, $P=.40)$ and accuracy $(90.2 \%$ compared with $88.1 \%, P=.59$ ) compared with fluoroscopy; these increases were not significant.

There were no biopsy-related serious complications including hemorrhage, infection, sinus tract formation, fracture, or pneumothorax in our study. Therefore, fluoroscopy-guided percutaneous spine biopsy is thought to be a safe procedure.

Because our study was retrospective, total procedure time was 

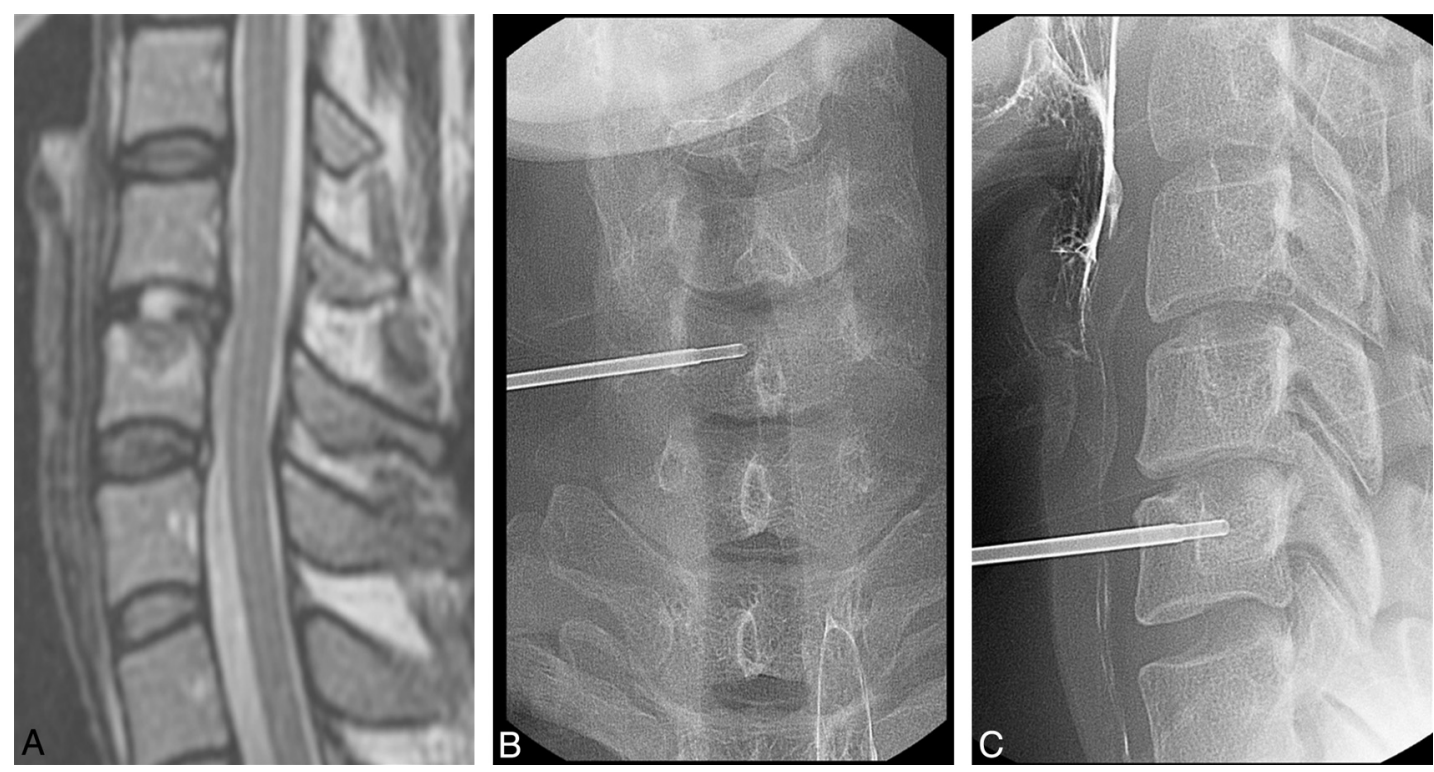

FIG 1. A 35-year-old woman with neck pain and a sense of heat in both shoulders for 6 months. A, Sagittal T2-weighted MR image shows low signal change with upper endplate destruction at the C6 upper body. Percutaneous biopsy was performed at the C 6 upper body with a right unilateral anterior approach under fluoroscopic guidance, which is seen on anteroposterior $(B)$ and lateral $(C)$ spot radiographs. Biopsy was confirmed as chronic osteomyelitis, but the causative organism was not isolated.
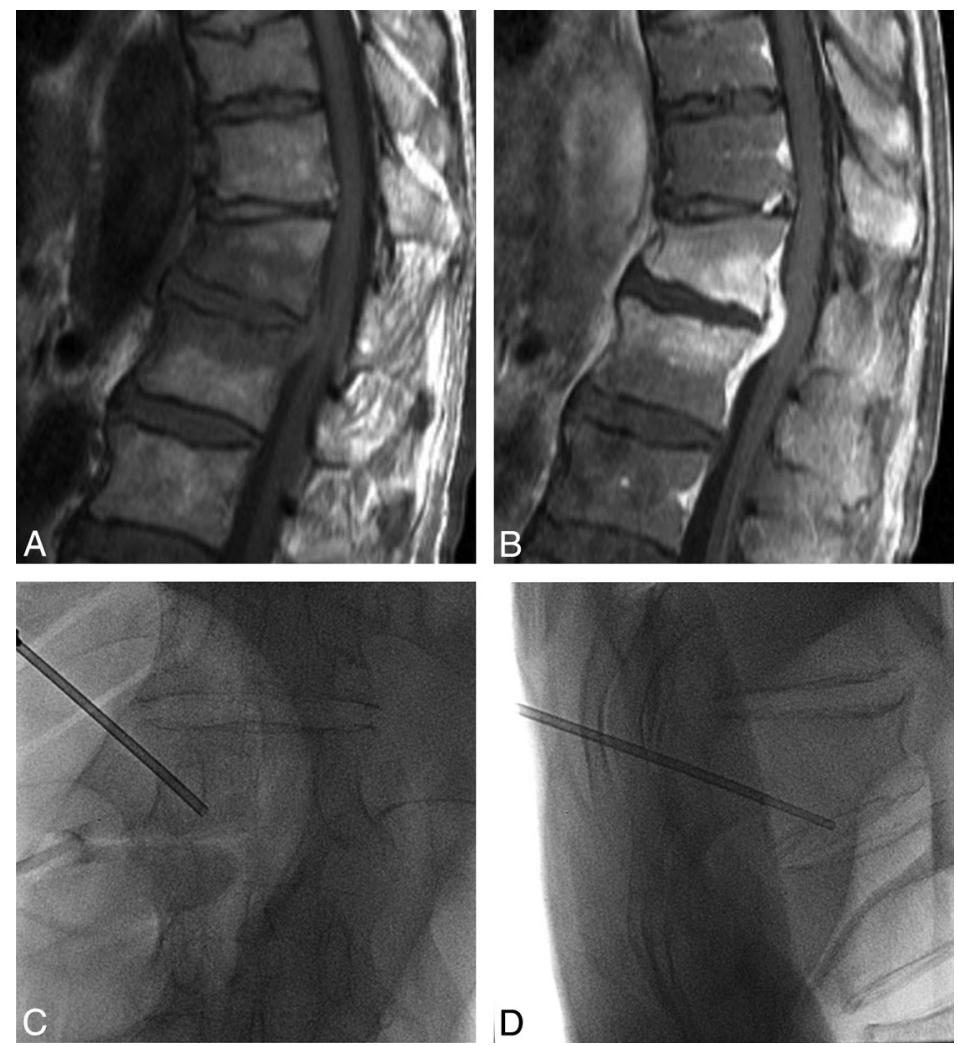

FIG 2. A 69-year-old man with fever 15 days after an operation for mechanical obstruction. Sagittal T1-weighted (A) and enhancement (B) MR images show low bone marrow signal change with enhancement at $\mathrm{T} 12$ and the L1 body and abscess formation at the anterior epidural space of the T12-L1 level. Percutaneous biopsy was performed at the T12 lower body with a left unilateral transpedicular approach under fluoroscopic guidance, which is demonstrated on anteroposterior $(C)$ and lateral $(D)$ spot radiographs. Pathologic findings were consistent with infectious spondylitis. The isolated causative organism was Klebsiella pneumoniae.

not recorded. However, in practice, procedure time took $15 \mathrm{~min}$ utes on average and did not exceed 20 minutes.

Percutaneous spine biopsy has been recommended for isolating causative organisms. However, the reported accuracy of spinal biopsy varies from $36 \%$ to $76.5 \%$, depending on the causative organism isolated. ${ }^{10,11,29-32}$ D'Agostino et al ${ }^{29}$ classified cases of spondylodiskitis into 2 groups, 38 cases being community-acquired and 43 cases hospital-acquired. A causative organism for 

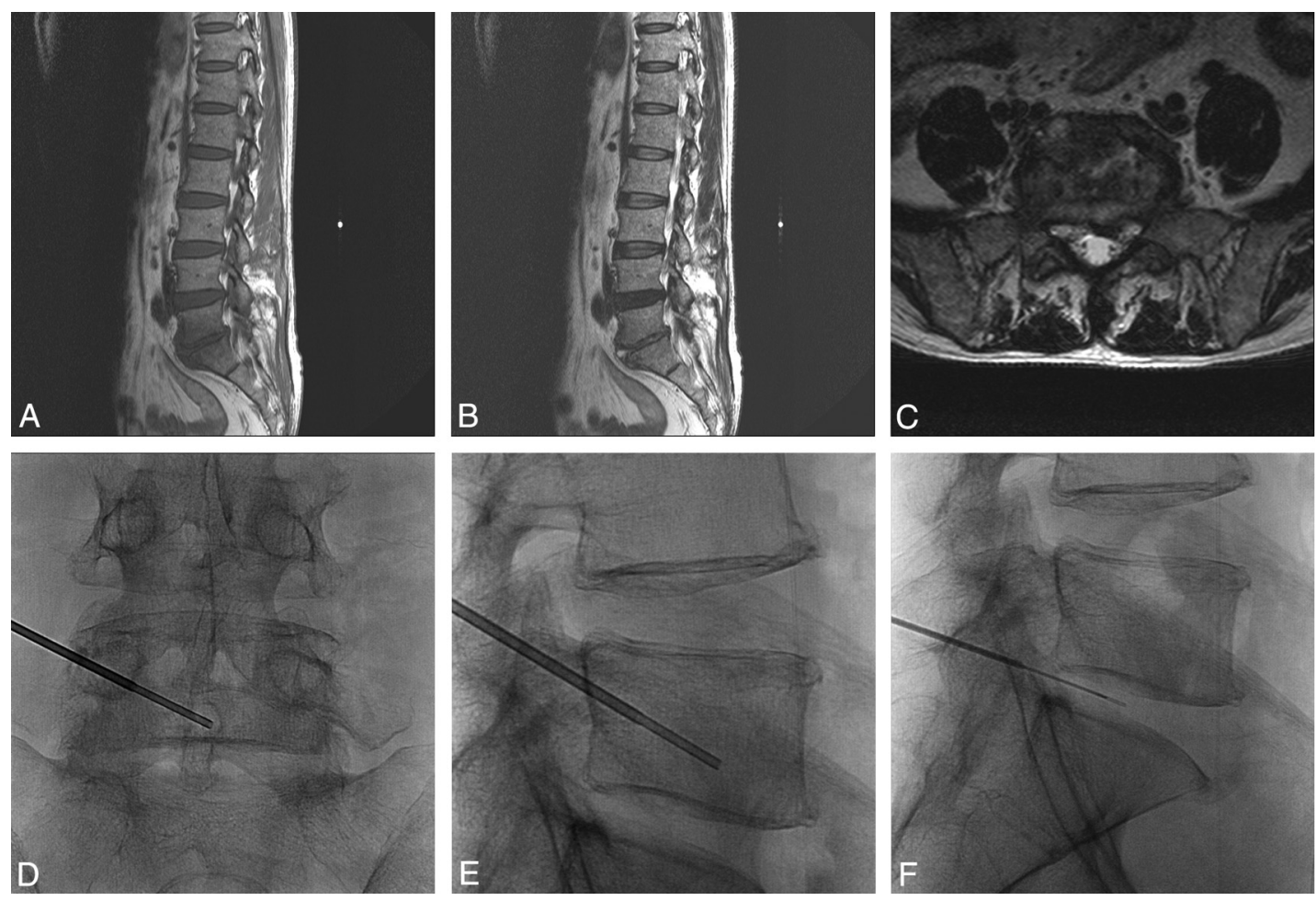

FIG 3. A 53-year-old man with low back pain and a chilling sensation for 1 week. Sagittal T1- (A), T2- (B), and axial T2-weighted (C) MR images show $\mathrm{T} 2$ high-fluid signal intensity in the L5/S1 disk space with T7 low marrow signal change in the peripheral portion of the L5 lower and S1 upper bodies and paravertebral extension. As seen on anteroposterior $(D)$ and lateral $(E$ and $F)$ spot radiographs, percutaneous biopsy and disk aspiration were performed at the left inferior lower body of the L5 vertebra with a left unilateral transpedicular approach under fluoroscopic guidance. Biopsy confirmed infectious spondylitis, but the causative organism was not isolated. After empiric antibiotic treatment, clinical symptoms were improved.

spondylodiskitis was isolated in $62(76.5 \%)$ patients. In a retrospective study of postoperative spondylodiskitis, Fouquet et $\mathrm{al}^{30}$ obtained a positive bacteriologic diagnoses in $9(36 \%)$ of 25 patients who had biopsies. Rankine et $\mathrm{al}^{10}$ analyzed 20 patients for spinal infections by percutaneous spinal biopsy. They isolated organisms in 6 of 12 patients not taking antibiotics, but in only 2 of 8 patients taking antibiotics. Yang et $\mathrm{al}^{32}$ performed CT-guided biopsy to diagnose cases of suspected spine infection and identified causative pathogens in 15 (47\%) of 32 biopsy specimens. In our study, the causative organisms were identified in 51 of 134 patients $(38.1 \%)$. Compared with previous studies by using CT guidance, our study is within the reported range.

In this study, re-biopsy due to failure to culture the causative organism on initial biopsy showed negative results in all except 2 patients. Thus, we recommend against rebiopsy for culture-negative patients.

The limitations of this study include the following: First, because our study was a retrospective one, there is the possibility of selection bias. Second, several clinical variables influencing the results of our study could not be controlled, such as prognostic factor, underlying disease such as diabetes mellitus or hypertension, previous operation history, and so forth. Clinical outcomes and long-term follow-up between the groups with and without identified causative organisms could not be compared. However, the main focus of our study was to evaluate the usefulness of fluoroscopic guidance for percutaneous spine biopsy in infectious spondylitis, so this information was deemed irrelevant.

In conclusion, fluoroscopy-guided percutaneous spine biopsy is a safe and accurate procedure and can be a preferred guidance method for diagnosis of infectious spondylitis.

\section{REFERENCES}

1. Kim SW, Lee SM, Shin H. Preoperative gadolinium-enhanced magnetic resonance images on infectious spondylitis. J Korean Neurosurg Soc 2005;38:355-58

2. Cottle L, Riordan T. Infectious spondylodiscitis. J Infect 2008;56:401-12

3. Rimondi E, Staals EL, Errani C, et el. Percutaneous CT-guided biopsy of the spine: results of 430 biopsies. Eur Spine J 2008;17:975-81

4. Renfrew DL, Whitten CG, Wiese JA, et el. CT-guided percutaneous transpedicular biopsy of the spine. Radiology 1991;180:574-76

5. Chew FS, Kline MJ. Diagnostic yield of CT-guided percutaneous aspiration procedures in suspected spontaneous infectious diskitis. Radiology 2001;218:211-14

6. Michel SC, Pfirrmann CW, Boos N, et el. CT-guided core biopsy of subchondral bone and intervertebral space in suspected spondylodiskitis. AJR Am J Roentgenol 2006;186:977-80

7. Lis E, Bilsky MH, Pisinski L, et el. Percutaneous CT-guided biopsy of osseous lesion of the spine in patients with known or suspected malignancy. AJNR Am J Neuroradiol 2004;25:1583-88

8. Cronin CG, Cashell T, Mhuircheartaigh JN, et el. Bone biopsy of new suspicious bone lesions in patients with primary carcinoma: prevalence and probability of an alternative diagnosis. AJR Am J Roentgenol 2009;193:W407-10

9. Dave B, Nanda A, Anandjiwala J. Transpedicular percutaneous biopsy of vertebral body lesions: a series of 71 cases. Spinal Cord 2009;47:384-89

10. Rankine JJ, Barron DA, Robinson P, et el. Therapeutic impact of percutaneous spinal biopsy in spinal infection. Postgrad Med J 2004;80:607-09 
11. Staatz G, Adam GB, Keulers P, et el. Spondylodiskitic abscesses: CTguided percutaneous catheter drainage. Radiology 1998;208:363-67

12. Kowalski TJ, Huddleston PM, Steckelberg JM, et el. Do follow-up imaging examinations provide useful prognostic information in patients with spine infections? Clin Infect Dis 2006;43:172e9

13. Butler JS, Shelly MJ, Timlin M, et el. Nontuberculous pyogenic spinal infection in adults: a 12-year experience from a tertiary referral center. Spine (Phila Pa 1976) 2006;31:2695-700

14. Pierot L, Boulin A. Percutaneous biopsy of the thoracic and lumbar spine: transpedicular approach under fluoroscopic guidance. $A J N R$ Am J Neuroradiol 1999;20:23-25

15. Maiuri F, Iaconetta G, Gallicchio B, et el. Spondylodiscitis: clinical and magnetic resonance diagnosis. Spine (Phila Pa 1976) 1997;22:1741-46

16. Acosta FL Jr, Galvez LF, Aryan HE, et el. Recent advances: infections of the spine. Curr Infect Dis Rep 2006;8:390-93

17. O'Daly BJ, Morris SF, O'Rourke SK. Long-term functional outcome in pyogenic spinal infection. Spine (Phila Pa 1976) 2008;33:E246-53

18. McHenry MC, Easley KA, Locker GA. Vertebral osteomyelitis: longterm outcome for 253 patients from 7 Cleveland-area hospitals. Clin Infect Dis 2002;34:1342-50

19. An HS, Seldomridge JA.. Spinal infections: diagnostic tests and imaging studies. Clin Orthop Relat Res 2006;444:27-33

20. Rezai AR, Woo HH, Errico TJ, et al. Contemporary management of spinal osteomyelitis. Neurosurgery 1999;44:1018-25

21. Robertson RC, Ball RP. Destructive spine lesions: diagnosis by needle biopsy. J Bone Joint Surg 1935;57-A:749-58
22. Craig, FS. Vertebral body biopsy. J Bone Joint Surg 1956;38-A:93-102

23. Ottolenghi CE. Aspiration biopsy of the spine. J Bone Joint Surg 1969;51-A:1531-43

24. Siffert RS, Arkin AM. Trephine biopsy of bone with special reference to the lumbar vertebral bodies. J Bone Joint Surg 1949;31-A:146-49

25. Jamshidi K, Swaim WR. Bone biopsy with unaltered architecture: a new biopsy device. J Lab Clin Med 1971;77:335-42

26. Wohlenberg H, Lorbacher P, Drechsel U, et al. Percutaneous punch biopsy of vertebral bodies with Jamshidi's needle [in German]. Dtsch Med Wochenschr 1978;103:902-04

27. Adapson BD, Lequda BD Jr, Lim EV, et el. CT-guided closed biopsy of the spine. J Comput Assist Tomogr 1981;5:737-38

28. Nourbakhsh A, Grady JJ, Garges KJ. Percutaneous spine biopsy: a meta-analysis. J Bone Joint Surg Am 2008;90:1722-25

29. D'Agostino C, Scorzolini L, Massetti AP, et el. A seven-year prospective study on spondylodiscitis: epidemiological and microbiological features. Infection 2010;38:102-07

30. Fouquet B, Goupille P, Jattiot F, et el. Discitis after lumbar disc surgery: features of "aseptic" and "septic" forms. Spine (Phila Pa 1976) 1992;17:356-58

31. Parker LM, McAfee PC, Fedder IL, et el. Minimally invasive surgical techniques to treat spine infections. Orthop Clin North Am 1996;27:183-99

32. Yang SC, Fu TS, Chen $\mathrm{LH}$, et el. Identifying pathogen of spondylodiscitis: percutaneous endoscopy or CT-guided biopsy. Clin Orthop Relat Res 2008;466:3086-92 